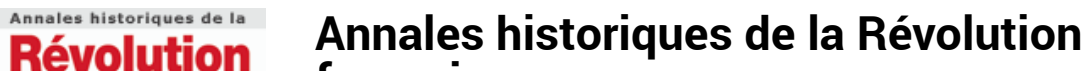

française française

352 | avril-juin 2008

Les temps composés de l'économie

\section{Serge Aberdam, Démographes et démocrates. L'œuvre} du Comité de division de la Convention nationale

\section{Malcolm Crook}

\section{(2) OpenEdition}

1 Journals

\section{Édition électronique}

URL : https://journals.openedition.org/ahrf/11036

DOI : 10.4000 /ahrf.11036

ISSN : 1952-403X

Éditeur :

Armand Colin, Société des études robespierristes

\section{Édition imprimée}

Date de publication : 1 juin 2008

Pagination : 294-296

ISBN : 978-2200-92514-7

ISSN : 0003-4436

Référence électronique

Malcolm Crook, «Serge Aberdam, Démographes et démocrates. L'œuvre du Comité de division de la Convention nationale », Annales historiques de la Révolution française [En ligne], 352 | avril-juin 2008, mis en ligne le 18 décembre 2009, consulté le 23 avril 2022. URL : http://journals.openedition.org/ahrf/ 11036 ; DOl : https://doi.org/10.4000/ahrf.11036

Ce document a été généré automatiquement le 23 avril 2022.

Tous droits réservés 


\title{
Serge Aberdam, Démographes et démocrates. L'œuvre du Comité de division de la Convention nationale
}

\author{
Malcolm Crook
}

\section{RÉFÉRENCE}

Serge Aberdam, Démographes et démocrates. L'œuvre du Comité de division de la Convention nationale, Paris, Société des études robespierristes, 2004, 391 p., ISBN 2-908327-50-3, $50 €$

1 Couronné du prix Albert Mathiez en 2002, la grande thèse de Serge Aberdam, soutenue à l'Université de Paris I, est présentée en partie dans ce livre extrêmement riche et suggestif. Ce volume reprend la première partie de son étude de l'élargissement du vote entre 1792 et 1795. Au travers du formidable travail du Comité (d'abord section) de division, on découvre des liens intimes entre démographie et démocratie, deux termes qui partagent une racine grecque commune : «demos». Sous la Révolution, en effet, le principe de la souveraineté du peuple, exercé dans la participation fréquente des ayants droit de voter aux élections, pousse le gouvernement français vers une connaissance plus exacte de sa population. Certes, toujours sensible aux significations des mots, Serge Aberdam nous rappelle que le terme " démographie » n'est pas inventé avant le milieu du XIX ${ }^{e}$ siècle, et nous met également en garde dans l'usage de «statistique », et même de "démocratie ». Un apprentissage de la démographie et de la démocratie résulte cependant des efforts administratifs et politiques des années 1790 , et représente une évolution fondamentale que Serge Aberdam souligne ici.

2 Son livre se compose de trois grandes sections, avec beaucoup de subdivisions pour guider le lecteur car, comme dans la thèse, l'argumentation est détaillée et demande mais aussi récompense énormément - une lecture attentive, voire des relectures. La grande enquête entreprise sous la Convention se trouve naturellement au cœur du 
travail et prend plus de la moitié du texte. Pourtant, dans un premier temps, Serge Aberdam considère les origines des dénombrements révolutionnaires et les essais tâtonnants de la Constituante pour connaître l'importance de la population en même temps que celle des citoyens actifs. Vers la fin du volume, il examine cette fois le cheminement des connaissances sous la Convention; sans avoir de grande application immédiate, elles exercent cependant une influence importante sous le Directoire et le Consulat.

3 Serge Aberdam commence par des regards critiques sur les dénombrements du XVIII siècle car - et c'est un aspect des Lumières - on commence à s'intéresser a l'arithmétique politique, y compris au nombre des habitants d'un pays. Cette science reste cependant inexacte, et les chiffres publiés par l'abbé Expilly, entre autres, laissent beaucoup à désirer. L'utilisation d'un coefficient conserverait pourtant longtemps ses partisans, mais la section de division (du Comité de constitution - on doit attendre l'ouverture de l'Assemblée législative pour la création d'un comité proprement dit) a le mérite de demander des dénombrements de la part des nouvelles autorités locales dans un contexte où nombre d'habitants et représentation politique vont ensemble. Mais, peut-on avoir confiance dans ces chiffres d'ayants droit de voter et de population? En effet, la correspondance entre citoyens actifs et niveau de population est bien variable, car localement déterminée. Comme l'auteur l'explique bien, le suffrage restreint de ces premières années de la Révolution reste un enjeu politique et tout essai d'emploi d'un multiplicateur à partir de listes de votants est voué à l'échec. En passant par une excellente discussion des modalités d'inscription électorale - citoyens "négligents ", aussi bien que "passifs» - Serge Aberdam accepte la réévaluation récente de la population française à 28 millions et, par conséquent, ajuste la correspondance avec les ayants droit de voter.

4 Sa reconstitution minutieuse de l'enquête sur la population et le droit de vote entreprise sous la Convention met en valeur, avant tout, les efforts méconnus des révolutionnaires afin de mieux cerner ces données fondamentales. Après avoir remarqué le peu de différence pratique apporté par l'abolition du paiement de trois journées de travail en impôts locaux dans les élections d'août 1792, l'auteur passe à l'établissement d'un nouveau Comité de division, dont les pouvoirs prendront une importance accrue. La décision de fonder la représentation nationale sur la seule population donne une impulsion au projet de dénombrement. Cette tâche devient plus urgente quand on inscrit l'élection directe des députés nationaux par circonscription de 39000 à 41000 citoyens, dans la célèbre Constitution de 1793. La prochaine convocation des assemblées primaires, à la suite de son acceptation par le peuple, demande des données démographiques plus sûres. On fait de plus grands et plus fiables efforts, appuyés par le gouvernement révolutionnaire à Paris et, au niveau local, secondés par les administrations de district. L'enquête continue jusqu'au printemps de 1795, quand l'abandon de la mise en activité de la Constitution «montagnarde » fait avorter le projet (les élections seront encore une fois indirectes et le suffrage de nouveau restreint). Ce travail d'Hercule du comité fait ressortir des données remarquables, nettement supérieures à celles de 1790-1791: une population de 28 millions d'habitants et des taux d'ayants droit de voter souvent proches de $28 \%$. Pourtant, faute de publication immédiate, ce résultat exceptionnel serait injustement négligé. 
5 En effet, ces renseignements sont en partie utilisés dans les assemblées primaires et les élections législatives de septembre 1795, et pour établir les tribunaux correctionnels à la même époque. En fin de compte, ils contribuent à la création des arrondissements de 1800 (qui serviront de circonscriptions électorales sous Napoléon). Le labeur des enquêteurs n'est pas complètement perdu, et il sera également exploité par l'archiviste Camus et le géographe Belleyme, tous deux associés au comité. Malgré l'absence de la publication prévue, les taux obtenus influencent la controverse continue autour de la méthodologie démographique - dénombrement ou coefficient - dont témoigne l'édition, en 1798, des deux belles cartes, assorties de chiffres départementaux de population, qui embellissent la couverture du livre d'Aberdam. En l'absence d'une direction centrale, la formation des listes des ayants droit de voter dépend de la bonne volonté des autorités locales (les districts disparus) et redevient donc bien variable. C'est l'avènement de Bonaparte qui marque la naissance d'une présentation officielle et comparative des «statistiques » dans ce domaine. Le nouveau régime commence par la proclamation d'un vote manipulé de la consultation populaire sur la Constitution de l'an VIII, avant d'organiser des recensements de population avec l'essai de 1800-1801, suivi de l'effort mieux réussi et bien connu de 1806. Encore une fois, ce travail démographique est lié aux effectifs des ayants droit de voter, d'un suffrage masculin de nouveau élargi.

6 Ces dernières données, comme le discrédit des calculs par multiplicateurs, doivent beaucoup aux efforts précédents de 1793-1795, injustement tombés dans l'oubli jusqu'à ce livre. Serge Aberdam démontre ainsi que, malgré la réticence de beaucoup d'administrations locales à élargir le suffrage, on y arrive finalement avec quelque sept millions d'ayants droit de voter en 1795. L'ironie de ce grand pas en avant est qu'il coïncide avec un reflux politique, qui rattache un critère fiscal à l'exercice du vote. Néanmoins, sous le Directoire, les effectifs de votants sont toujours plus nombreux qu'aux débuts de la Révolution. Certes, à aucun moment on ne peut parler de suffrage universel, même pour les hommes adultes, ce qui explique la préférence pour l'expression «élargissement du vote». Pourtant, des zones apparaissent sur la cartographie, comme le môle champenois, où les taux des ayants droit de voter sont plus élevés que la moyenne. Cette géographie politique est difficile à expliquer mais, dans la Champagne, elle est aussi associée à un relativement fort niveau de participation électorale. La production, sous l'égide du Comité de division, d'un « cadastre des âmes » révèle ainsi les liens entre démographie et démocratie, toutes les deux étant en nette progression sous la Révolution.

7 Serge Aberdam excelle à la contextualisation des sources primaires et secondaires, avec ses aperçus profonds sur le travail des contemporains des années 1790, aussi bien que son analyse critique de la recherche historique (l'influence de Jacques Dupâquier, qui a conduit à la surévaluation des efforts napoléoniens et à la sous-estimation du grand projet du Comité de division). Sa reconstruction des archives dans les séries DIVbis ou F20 des Archives nationales est une superbe démonstration de sa propre technique. Son étude nous renseigne, de plus, sur le fonctionnement de l'administration au niveau local, aussi bien que sur les relations entre centre et localité. La biographie y trouve aussi une place avec ses remarques sur la carrière intéressante du député Antoine Mailly, principal animateur du comité ; l'auteur est en même temps attentif au profil de ses collègues. Son érudition lui permet de nous renseigner sur maints aspects de la démographie et de la démocratie : politiques, administratifs, intellectuels et culturels. 
Dans ce livre, il éclaire enfin l'histoire générale de la Révolution, comme ses communications et interventions aux colloques et réunions nous l'on déjà démontré.

8 Son texte est accompagné d'une trentaine de cartes et de figures, reproduites de superbe façon. Dans les annexes, on retrouve un excellent commentaire sur les sources documentaires consultées dans plus d'une vingtaine d'archives départementales et de nombreuses bibliothèques, aussi bien qu'aux Archives nationales. On y trouve également les circulaires du Comité de division, mais, malheureusement, pas les chiffres départementaux auxquels le comité est arrivé. On peut également regretter quelques oublis dans la bibliographie, et on n'y retrouve pas toujours les ouvrages qui apparaissent dans les notes d'une manière parfois insuffisamment détaillée. Au-delà de ces détails, le livre représente une contribution essentielle aux études électorales de la Révolution qui, ces dernières années, ont fait des progrès énormes. Serge Aberdam a d'ailleurs été pour beaucoup dans ce renouveau, qu'il a encouragé, et tous ceux qui travaillent ce champ de recherche savent ce qu'ils lui doivent. On ne peut qu'espérer que la publication de la deuxième partie de sa thèse, consacrée à la pratique de l'électorat élargi dans les votes populaires sur les Constitutions de 1793 et de 1795, puisse intervenir bientôt. 